\title{
Inter-rater agreement among shoulder surgeons on treatment options for proximal humeral fractures among shoulder surgeons
}

\author{
Hyojune Kim ${ }^{1}$, Si-Jung Song ${ }^{2, *}$, In-Ho Jeon ${ }^{2}$, Kyoung Hwan Koh ${ }^{2}$ \\ ${ }^{1}$ Department of Orthopedic Surgery, Eulji University Hospital, Daejeon, Korea \\ ${ }^{2}$ Department of Orthopedic Surgery, Asan Medical Center, University of Ulsan College of Medicine, Seoul, Korea
}

\begin{abstract}
Background: The treatment approach for proximal humeral fractures is determined by various factors, including patient age, sex, dominant arm, fracture pattern, presence of osteoporosis, preexisting arthritis, rotator cuff status, and medical comorbidities. However, there is a lack of consensus in the literature regarding the optimal treatment for displaced proximal humeral fractures. This study aimed to assess and quantify the decision-making process for either conservative or surgical treatment and the choice of surgical method among shoulder surgeons when treating proximal humeral fractures.

Methods: Forty sets of true anteroposterior view, scapular Y projection view, and three-dimensional computed tomography of proximal humeral fractures were provided to 12 shoulder surgeons along with clinical information. Surveys regarding Neer classification, decisions between conservative and surgical treatments, and chosen methods were conducted twice with an interval of 2 months. The factors affecting the treatment plans were also assessed.

Results: The inter-rater agreement was fair for Neer classification (kappa=0.395), moderate for the decision between conservative and surgical treatments $(\mathrm{kappa}=0.528)$, and substantial for the chosen method of surgical treatment $(\mathrm{kappa}=0.740)$. The percentage of agreement was $71.1 \%$ for Neer classification, $84.6 \%$ for the decision between conservative and surgical treatment, and $96.4 \%$ for the chosen method of surgical treatment. The fracture pattern was the most crucial factor in deciding between conservative and surgical treatments, followed by age and physical activity.

Conclusions: The decision between conservative and surgical treatment for proximal humeral fractures showed good agreement, while the chosen method between osteosynthesis and arthroplasty showed substantial agreement among shoulder surgeons.
\end{abstract}

Keywords: Proximal humeral fracture; Neer classification; Treatment option; Inter-rater agreement; Intra-rater agreement

\section{INTRODUCTION}

Proximal humeral fractures are the third most common osteopo- rotic fractures in elderly patients after spine and wrist fractures [1]. Although most fractures can be treated conservatively with good clinical results [2,3], complex fractures may require surgical

Received: July 1, $2021 \quad$ Revised: August 31, $2021 \quad$ Accepted: September 5, 2021

Correspondence to: Kyoung Hwan Koh

Department of Orthopedic Surgery, Asan Medical Center, University of Ulsan College of Medicine, 88 Olympic-ro 43-gil, Songpa-gu, Seoul 05505, Korea Tel: +82-2-3010-3530, Fax: +82-2-488-7877, E-mail: osdoc.koh@gmail.com, ORCID: https://orcid.org/0000-0002-6181-9621

${ }^{*}$ Current affiliation: Department of Orthopaedic Surgery, Bundang Chuck Hospital, Seongnam, Korea

Financial support: None.

Conflict of interest: None.

Copyright@ 2022 Korean Shoulder and Elbow Society.

This is an Open Access article distributed under the terms of the Creative Commons Attribution Non-Commercial License (http://creativecommons.org/licenses/by-nc/4.0/) which permits unrestricted non-commercial use, distribution, and reproduction in any medium, provided the original work is properly cited. 
treatment to achieve anatomic alignment, early mobilization, and functional recovery. For surgical stabilization of these fractures, the use of a locking plate is gaining attention. Although clinical and radiological results are promising with locking plate fixation [4], the relatively high complication rate with this method can make the surgical stabilization of proximal humeral fractures in an osteoporotic bone challenging [5].

The treatment approach for proximal humeral fractures is determined by various factors, including patient age, sex, dominant arm, fracture pattern, presence of osteoporosis, preexisting arthritis, rotator cuff status, and medical comorbidities [6]. The severity of the fracture itself is the single most important determinant of the choice of intervention. However, even Neer classification of proximal humeral fractures appears to show low inter-rater reliability in most studies [7-10]. Moreover, similar fracture patterns can be treated differently according to the preference or experience of the treating surgeon [11-13]. Even for displaced proximal humeral fractures in elderly patients, there currently exists a wide variation in the method of surgical treatment, such as arthroplasty or osteosynthesis. As the incidence of osteoporotic fractures is increasing in aged populations, there is poor consensus on the optimal treatment for displaced proximal humeral fractures in the literature. Therefore, a study of current practice patterns is essential because these are important public health issues, and the results of such an investigation would be very helpful to surgeons for future treatment planning.

Therefore, the purpose of this study was to assess and quantify the decision-making process for either conservative or surgical treatment and the choice of surgical methods among shoulder surgeons when treating proximal humeral fractures. The researchers hypothesized that there would be variability of more than $10 \%$ regarding the decision to use surgical or conservative treatment among surgeons.

\section{METHODS}

The study was approved by the Institutional Review Board of Inje University Ilsan Paik Hospital (IRB No. 2017-05-002). An agreement of the patient was exempted as it was a retrospective study. The data of patients who visited the emergency room and outpatient clinic of a single institution from March 1, 2014, to July 31, 2016, and were diagnosed with a proximal humeral fracture were consecutively collected and retrospectively reviewed. Among them, 118 patients aged 18 years and above were independently evaluated by an assessor who was not involved in their diagnosis or treatment.

Sample size calculations revealed 40 cases showing 10\% in- ter-rater variability in terms of surgical and conservative treatment choices among raters, assuming a 5\% significance level and $80 \%$ power. Forty sets of true anteroposterior (AP) view, scapular $\mathrm{Y}$ projection view, and three-dimensional (3D) computed tomography (CT), which could indicate the detailed fracture patterns, were selected via general consensus of the authors to represent the full spectrum of proximal humeral fracture patterns from the 118 eligible patients. In cases of fracture dislocation, both initial and post-reduction radiographs were provided. For the selected 40 sets, medical records were reviewed for factors that could affect treatment decisions, including age, sex, injury mechanism, rheumatic disease, dementia, and medical comorbidities. All patient information other than clinical data was removed.

Twelve fellowship-trained shoulder surgeons who practiced in different university hospitals and had at least 5 years of experience were invited to participate in the study. Multiple-choice questions related to diagnosis and treatment for all 40 study subjects were surveyed, with information regarding clinical parameters including age, sex, injury mechanism, and medical comorbidities. A copy of Neer classification of proximal humeral fractures was also provided as a reference to all of the participating surgeons. The principal investigator for this study did not participate in the questionnaire survey.

The true AP view, scapular Y view, and 3D CTs were saved as DICOM files in the PACS system (M-view 5.4; Marotech, Seoul, Korea) (Fig. 1). For the cases with a fracture dislocation, the post-reduction true AP view and scapular Y view CTs were added as well. The raters were allowed to observe the radiographic data using RadiAnt DICOM viewer 4.0.2 (32 bit; Medixant, Poznan, Poland). The surveys were repeated 2 months later using a different ordering of the patients to assess for any differences with the same rater. All 12 participating surgeons completed the first survey, and nine completed both surveys.

Each rater answered the following questions for each of the 40 proximal humeral fractures in the study cohort: (1) What is the Neer classification? (2) Will you perform surgical or conservative treatment? (3) Which of the following factors are the two most important in determining whether to use surgical or conservative treatment? (a) Age and activity level; (b) fracture severity; (c) osteoporosis; (d) medical comorbidity; or (e) arm dominance. For the patients for whom surgical treatment was chosen, the following questions were asked: (1) What specific surgical procedures would you choose? (a) Closed reduction (CR) and pinning; (b) CR and intramedullary (IM) nail fixation; (c) osteosynthesis using plates and screws; (d) hemiarthroplasty; (e) reverse total shoulder arthroplasty; (f) minimally invasive plate osteosynthesis 

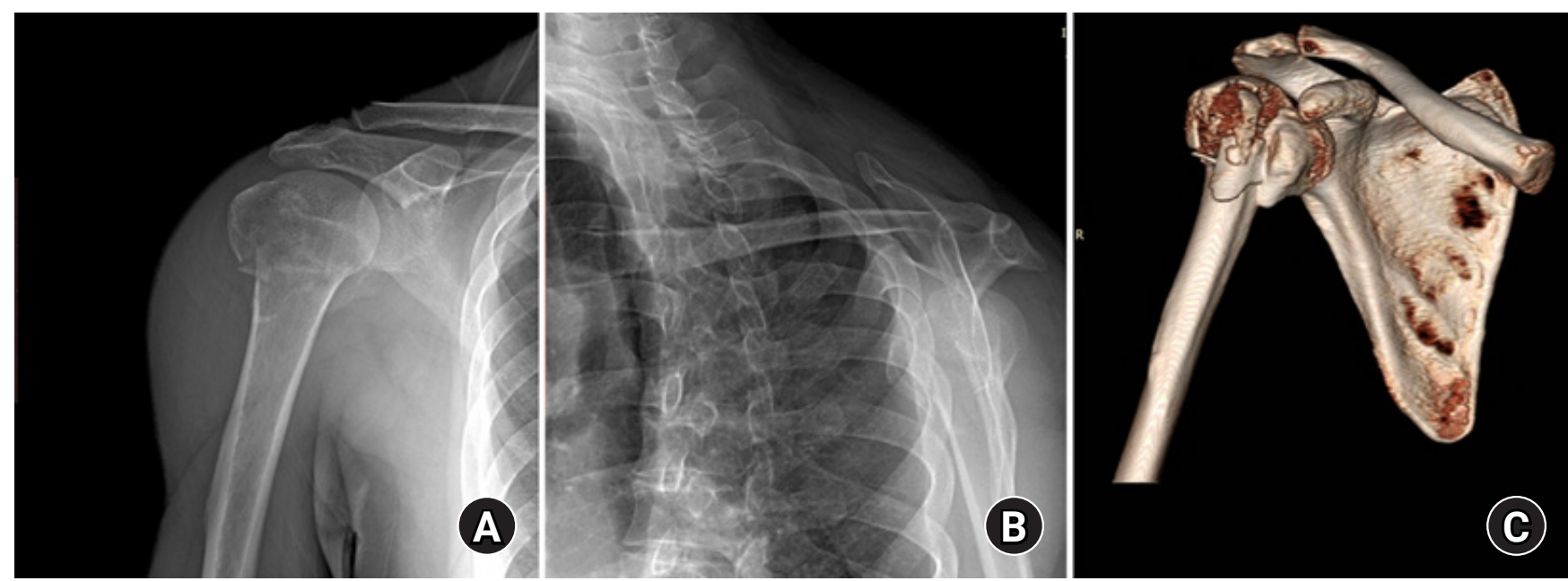

Fig. 1. Typical plain radiographs showing significant variations among the participating surgeons in terms of Neer classification. (A) True anteroposterior view. (B) Trans-scapular Y projection view. (C) Three-dimensional computed tomography.

(MIPO); or (g) arthroscopic fixation. (2) How long would you immobilize the patient after surgery? (3) Which immobilization method would you apply after surgery? (4) What are the two most important factors in determining the surgical method? (a) Age and activity level; (b) fracture severity; (c) osteoporosis; (d) medical comorbidity; or (e) cuff status.

If conservative treatment was chosen, the following questions were asked: (1) Which immobilization method would you apply? (2) When would you conduct your next follow-up radiographic examination? (3) For how long would you immobilize the patient? Specific instructions for determining the need for surgery were not provided to the raters, and they were allowed to use the same criteria that they use in their own clinical practice. The demographic information of each rater, including age, sex, and years of practice since fellowship training, was collected and analyzed.

\section{Statistical Analysis}

The inter-rater agreement concerning each survey question, including Neer classification, treatment options, surgical methods, and immobilization methods, was evaluated. The inter- and intra-rater reliabilities using Fleiss kappa for each domain were analyzed using R ver. 3.4.0. (R Foundation for Statistical Computing, Vienna, Austria; https://www.r-project.org/) [14,15]. The generated kappa values were interpreted according to the guidelines of Landis and Koch (i.e., 0.01-0.20, slight agreement; 0.210.40 , fair agreement; $0.41-0.60$, moderate agreement; $0.61-0.80$, substantial agreement; and 0.81-0.99, almost perfect agreement). For the statistical analysis of surgical methods, the patients who were selected for surgery by more than one rater were chosen, and the surgical methods were roughly categorized as arthroplasty or osteosynthesis. The first radiological follow-up was analyzed after categorization into less than 2 weeks or 2 weeks. Among the variables, such as age, sex, injury mechanism, medical comorbidities (compromised cardiopulmonary system, renal failure, and stroke history), and Neer classification, analysis was performed to determine which factor would affect the treatment option (conservative or surgical treatment) using the Cochran-Mantel-Haenszel test for stratified categorical data.

\section{RESULTS}

A total of 12 surgeons participated in this study. All of them were male and had a subspecialty in shoulder surgery and a mean age of 43.4 years (range, 38-48 years). The mean duration of experience after fellowship training was 7.8 years (range, $4-12$ years). The 40 proximal humeral fracture cases selected retrospectively for the study survey comprised cases from 27 female and 13 male patients with a mean age of $64.7 \pm 17.8$ years. Neer classification of the study cohort indicated a one-part fracture in $16.0 \%$, twopart fracture in $52.9 \%$, three-part fracture in $21.3 \%$, and fourpart fracture in $9.8 \%$ of the patients.

With respect to Neer classification, the percentage of agreement was $71.1 \%$ in the first review and $76.9 \%$ in the second review. Inter-rater agreement was fair to moderate (kappa $=0.395$ in the first review and kappa $=0.417$ in the second review), while the intra-rater agreement was substantial $($ kappa $=0.744)($ Table 1$)$. With respect to treatment choices, the mean percentage of agreement was $84.6 \%$ in the first review and $86.4 \%$ in the second review. The inter-rater agreement was moderate (kappa $=0.528$ in the first 
Table 1. Inter-rater agreement and the kappa coefficient and intra-rater agreement for Neer classification

\begin{tabular}{|c|c|c|c|c|}
\hline \multirow{2}{*}{ Variable } & \multirow{2}{*}{ No. of patients (\%) } & \multicolumn{2}{|c|}{ Inter-rater agreement } & \multirow{2}{*}{$\begin{array}{c}\text { Intra-rater agreement } \\
\text { Kappa (mean) }\end{array}$} \\
\hline & & Mean percentage of agreement (range) & Fleiss' kappa & \\
\hline First review $^{\dagger}$ & & $71.1(41.7-100.0)$ & 0.395 & 0.744 \\
\hline 1 & $77(16.0)$ & & & \\
\hline 2 & $254(52.9)$ & & & \\
\hline 3 & $102(21.3)$ & & & \\
\hline 4 & $47(9.8)$ & & & \\
\hline Second review ${ }^{*}$ & & $76.9(44.4-100.0)$ & 0.417 & 0.744 \\
\hline 1 & $42(11.7)$ & & & \\
\hline 2 & $203(56.4)$ & & & \\
\hline 3 & $75(20.8)$ & & & \\
\hline 4 & $40(11.1)$ & & & \\
\hline
\end{tabular}

${ }^{*}$ Nine raters evaluated the intra-rater agreement; ${ }^{\dagger}$ Twelve surgeons participated; ${ }^{*}$ Nine surgeons participated.

Table 2. Inter-rater agreement and its kappa coefficient and intra-rater agreement for the decision between conservative and surgical treatment

\begin{tabular}{|c|c|c|c|c|}
\hline \multirow{2}{*}{ Variable } & \multirow{2}{*}{ No. of patients (\%) } & \multicolumn{2}{|l|}{ Inter-rater agreement } & \multirow{2}{*}{$\begin{array}{c}\text { Intra-rater agreement } \\
\text { Kappa (mean) }\end{array}$} \\
\hline & & Mean percentage of agreement (range) & Fleiss' kappa & \\
\hline First review & & $84.6(50.0-100.0)$ & 0.528 & 0.879 \\
\hline Conservative treatment & $186(38.8)$ & & & \\
\hline Surgical treatment & $294(61.3)$ & & & \\
\hline Second review & & $86.4(55.6-100.0)$ & 0.549 & 0.879 \\
\hline Conservative treatment & $144(40.0)$ & & & \\
\hline Surgical treatment & $216(60.0)$ & & & \\
\hline
\end{tabular}

${ }^{*}$ Nine raters evaluated intra-rater agreement.

review and kappa $=0.549$ in the second review), while the intra-rater agreement was almost perfect (kappa $=0.879)($ Table 2).

Seven of the 12 participating surgeons responded that fracture pattern or severity was the most crucial factor in deciding between conservative and surgical treatment for a proximal humeral fracture: of these seven surgeons, five regarded age and activity level as the second most important factors. The remaining five raters indicated age and activity level as the most crucial factors and the fracture pattern or severity as the second most important factor. One rater indicated that medical comorbidities were the second most important factor, while another rater listed the dominant arm as such.

When surgical treatment was chosen, $\mathrm{CR}$ and pinning was the method of choice in $3.1 \%$ of the patients, CR and IM nail fixation in $9.9 \%$, open reduction internal fixation in 59.5\%, hemiarthroplasty in $3.4 \%$, reverse total shoulder arthroplasty in $15.6 \%$, MIPO in 5.1\%, and arthroscopic fixation in 3.4\%. When the surgical options were stratified into osteosynthesis and arthroplasty, 56 (19.05\%) out of 294 patients were chosen for arthroplasty and $238(80.95 \%)$ for osteosynthesis in the first review. The percentage of agreement was $96.4 \%$ in the first review and $96.2 \%$ in the second review. The inter-rater agreement was substantial for these reviews, with a mean kappa value of 0.740 and 0.727 , respectively.

All surgeons indicated that they would apply immobilization even after surgical treatment, for a mean of 4.2 weeks (median, 4 weeks; range, 2-6 weeks). In most cases, abduction brace application was the indicated method for immobilization (205 of 294 cases), with a mean percentage of agreement of $73.0 \%$. The second radiographic examination after the surgery would have been performed at 2 weeks, with a mean percentage of agreement of $76.5 \%$ or $70.4 \%$, although the kappa coefficient showed only a slight agreement (Table 3).

When conservative treatment was chosen, the surgeons indicated that the shoulders would have been immobilized using an arm sling in 5.9\%, a Velpeau sling in $21.5 \%$, an abduction brace in $65.6 \%$, and a splint in $7 \%$ of the patients. The suggested mean duration of immobilization for conservative treatment was slightly longer than that after surgical treatment, with a mean value of 4.7 weeks (median, 4 weeks; range, 2-8 weeks). The suggested mean time of the next radiographic follow-up was 8.6 days (median, 7 days; range, 3-14 days) (Table 4).

The study also investigated whether age, sex, injury mechanism, medical comorbidities (compromised cardiopulmonary 
Table 3. Inter-rater agreement for surgery

\begin{tabular}{|c|c|c|c|}
\hline \multirow{2}{*}{ Variable } & \multirow{2}{*}{ No. of patients (\%) } & \multicolumn{2}{|c|}{ Inter-rater agreement ${ }^{*}$} \\
\hline & & Mean percentage of agreement (range) & Fleiss' kappa \\
\hline \multicolumn{4}{|l|}{ Surgical option } \\
\hline First review & & $96.4(58.3-100.0)$ & 0.740 \\
\hline Arthroplasty & $56(19.0)$ & & \\
\hline Osteosynthesis & $238(81.0)$ & & \\
\hline Second review & & $96.1(66.7-100.0)$ & 0.727 \\
\hline Arthroplasty & $46(21.3)$ & & \\
\hline Osteosynthesis & $170(78.7)$ & & \\
\hline \multicolumn{4}{|l|}{ Immobilization method } \\
\hline First review & & $73.0(33.3-100.0)$ & $-^{\dagger}$ \\
\hline Arm sling & 35 (11.9) & & \\
\hline Velpeau sling & $53(18.0)$ & & \\
\hline Abduction brace & $205(69.7)$ & & \\
\hline Splint & $1(0.3)$ & & \\
\hline Second review & & $80.6(33.3-100.0)$ & $-^{\dagger}$ \\
\hline Arm sling & $7(3.2)$ & & \\
\hline Velpeau sling & $45(20.8)$ & & \\
\hline Abduction brace & $164(75.9)$ & & \\
\hline Splint & $1(0.5)$ & & \\
\hline \multicolumn{4}{|c|}{ Next radiographic evaluation (day) } \\
\hline First review & & $76.5(50.0-100.0)$ & 0.004 \\
\hline$<14$ & $71(24.1)$ & & \\
\hline 14 & $223(75.9)$ & & \\
\hline Second review & & $70.4(50.0-100.0)$ & -0.080 \\
\hline$<14$ & $64(29.6)$ & & \\
\hline 14 & $152(70.4)$ & & \\
\hline \multicolumn{4}{|c|}{ Immobilization period (wk) } \\
\hline First review & & $51.0(33.3-100.0)$ & $-^{\dagger}$ \\
\hline 2 & $55(18.7)$ & & \\
\hline 3 & $22(7.5)$ & & \\
\hline 4 & $120(40.8)$ & & \\
\hline 6 & $97(33.0)$ & & \\
\hline Second review & & $49.2(33.3-100.0)$ & $-{ }^{\dagger}$ \\
\hline 2 & $17(7.9)$ & & \\
\hline 3 & $23(10.6)$ & & \\
\hline 4 & $91(42.1)$ & & \\
\hline 5 & $9(4.2)$ & & \\
\hline 6 & $76(35.2)$ & & \\
\hline
\end{tabular}

${ }^{\star}$ The inter-rater agreement was analyzed only in cases where more than one rater selected the surgical treatment option; ${ }^{\dagger}$ The kappa coefficient could not be calculated.

system, renal failure, and stroke history), and fracture classification affected the choice of conservative or surgical treatment but only found a significant association with respect to Neer classification ( $\mathrm{p}<0.001$ ) (Table 5). The medical comorbidities could not be analyzed, and multivariate analysis could not be performed because of the small sample size.

\section{DISCUSSION}

The present study showed that the inter-rater agreement regarding treatment decisions and surgical options for proximal humeral fractures was moderate and substantial, respectively. The percentage of agreement for assessing the fracture type and treatment decisions ranged from $71.1 \%$ to $86.4 \%$. These findings sup- 
Table 4. Inter-rater agreement in cases of conservative treatment

\begin{tabular}{lcc}
\hline \multirow{2}{*}{ Variable } & No. of patients $(\%)$ & \multicolumn{2}{c}{ Inter-rater agreement $^{*}$} \\
\cline { 3 - 3 } Immobilization method & & Mean percentage of agreement (range) \\
$\quad$ Arm sling & $11(5.9)$ & \\
Velpeau sling & $40(21.5)$ & \\
Abduction brace & $122(65.6)$ & \\
Splint & $13(7.0)$ & $76.4(50.0-100.0)$ \\
Next radiographic evaluation (day) & & \\
$<14$ & $136(73.1)$ & \\
14 & $50(26.9)$ & $50.8(33.3-83.3)$ \\
Immobilization period (wk) & & \\
2 & $4(2.2)$ & \\
3 & $34(18.3)$ & \\
4 & $65(34.9)$ & \\
5 & $3(1.6)$ & \\
6 & $77(41.4)$ & \\
8 & $3(1.6)$ & \\
\hline
\end{tabular}

${ }^{*}$ The inter-rater agreement was analyzed only in cases where more than one rater selected the surgical treatment option; ${ }^{\dagger}$ The kappa coefficient could not be calculated.

Table 5. Correlation between Neer classification and treatment options

\begin{tabular}{lccc}
\hline Neer classification & $\begin{array}{c}\text { Conservative } \\
\text { treatment }\end{array}$ & $\begin{array}{c}\text { Surgical } \\
\text { treatment }\end{array}$ & p-value* \\
\hline 1 & $71(38.2)$ & $6(2.0)$ & $<0.001$ \\
2 & $100(53.8)$ & $154(52.4)$ & \\
3 & $15(8.1)$ & $87(29.6)$ & \\
4 & 0 & $47(16.0)$ & \\
\hline
\end{tabular}

Values are presented as number (\%).

* Statistical significance was tested using the Cochran-Mantel-Haenszel test.

port the study hypothesis that there would be variability of more than $10 \%$ among surgeons for the decision on the fracture type and treatment options.

Previous studies have already shown that the reproducibility or agreement for Neer classification of proximal humeral fractures is low. The kappa values in these studies ranged from somewhere between 0.183 and 0.633 using radiography and two-dimensional or 3D CT scans $[10,12,13,16,17]$. Thus, several alternative classifications have been introduced $[8,18,19]$. Nevertheless, Neer classification is still used in clinical practice because it provides convenience in terms of communication and overall guidelines for surgical indication.

The present study provided 12 specialist shoulder surgeons with 3D CTs and clinical information as well as plain radiographs for a retrospective cohort of 40 proximal humeral fracture cases.
These clinicians were then asked to determine Neer classification and advise on the treatment plans they would choose. As expected, inter-rater agreement with regard to Neer classification was similar to that reported in previous studies, with a mean kappa value ranging from 0.395 to 0.417 . The choice of surgical or conservative treatment showed a similarly low level of agreement as in previous reports. However, when the percentage of agreement for individual questions was evaluated, which is an intuitive way to perform a reliability test, relatively high levels of agreement were observed (i.e., a $71 \%$ level of agreement for Neer classification); this indicated that 8.5 out of the 12 (71\% of the 12$)$ surgeon raters agreed on Neer classification for the same fracture pattern, although the kappa statistics showed only a slight or fair reliability. Similarly, the percentage of agreement was $84 \%-86 \%$ for the choice of conservative versus surgical treatment, while the kappa statistics demonstrated moderate agreement (0.528-0.549). Furthermore, the inter-rater agreement for the surgical method, arthroplasty versus osteosynthesis, was very high (approximately $96 \%$ ) and substantial (with kappa values of 0.740 or 0.727 ). Contrary to what has been proposed in previous studies [7-13], these observations might suggest that there may be some consensus regarding the diagnosis and treatment of osteoporotic proximal humeral fractures. Even if there is a discrepancy in fracture classifications or the selection of conservative and surgical treatments, the choice of the surgical method between arthroplasty and osteosynthesis showed relatively high agreement among the 
surgeons in this study. Therefore, the treatment plan for these fractures could be similar among surgeons, even though the fracture pattern is assessed differently.

In the study survey, most of the surgeons preferred osteosynthesis over arthroplasty, even in complex cases, such as four-part fractures, although all 12 participating surgeons had a shoulder subspecialty. Reverse arthroplasty has gained more popularity recently, and some reports have indicated that this method can even be superior to osteosynthesis for treating fractures $[20,21]$. Notably however, it appears that most of the shoulder surgeons in this study weighed the benefits of a well-restored anatomy by osteosynthesis against the higher risk of complications with arthroplasty $[22,23]$.

In contrast to previous reports, this study found that osteoporosis and medical comorbidities were not regarded as significant factors for determining the treatment plans [24]. Okike et al. [24] reported that patients with medical comorbidities and osteoporosis commonly underwent surgical intervention for arthroplasty. However, all of the surgeons surveyed in the present study would have applied immobilization even after surgical treatment. The indicated duration of this immobilization was also similar to that for conservative treatments. This might suggest that even if osteoporosis were not a crucial consideration during treatment decision-making, all of the surgeons surveyed in the present study had concerns about a possible secondary reduction loss caused by osteoporosis. Most of them seemed to give greater weight to protection than early mobilization, even when choosing a surgical treatment.

Previous studies have reported good clinical outcomes with conservative treatment of proximal humeral fractures $[1,25]$ and that even displaced fractures can be treated successfully with a nonoperative approach [3]. All surgical options for proximal humeral fractures, including IM nailing, open osteosynthesis, and arthroplasty, have yielded favorable outcomes [26-30]. However, the currently available evidence from randomized controlled trials is insufficient to guide decision-making between different non-surgical and surgical interventions for these fractures [2]. The current study found substantial variabilities in fracture classification, treatment planning, surgical methods, and immobilization methods and duration. Thus, individually tailored treatment plans should be adopted with consideration for the fracture pattern but also patient-related factors and the surgeon's experience and preferences.

This study had certain limitations. The principal aim of the study was to determine the trends in decision-making for the treatment of proximal humeral fractures among shoulder surgeons. Thus, the specific treatment that the included patients ac- tually received was not investigated. Furthermore, the small number of patients and involved surgeons limited the power of the statistical analysis regarding the true factors affecting the choice of treatment. However, the researchers provided the participating surgeons with relevant clinical information on the retrospective cases, including age, sex, and medical comorbidities, in addition to radiographs and $3 \mathrm{D}$ CTs. This matches the information that would be available in an actual clinical setting.

Although Neer classification of proximal humeral fractures did not demonstrate high agreement among the treating surgeons, the choice of conservative versus surgical treatment showed good agreement. Furthermore, the choice between osteosynthesis and arthroplasty showed a substantial agreement among the shoulder surgeons.

\section{ORCID}

Hyojune Kim

https://orcid.org/0000-0001-7665-536X

Si-Jung Song

In-Ho Jeon

https://orcid.org/0000-0003-1215-5264

Kyoung Hwan Koh

https://orcid.org/0000-0002-9289-9193

https://orcid.org/0000-0002-6181-9621

\section{REFERENCES}

1. Court-Brown CM, Garg A, McQueen MM. The translated twopart fracture of the proximal humerus: epidemiology and outcome in the older patient. J Bone Joint Surg Br 2001;83:799-804.

2. Handoll HH, Brorson S. Interventions for treating proximal humeral fractures in adults. Cochrane Database Syst Rev 2015; (11):CD000434.

3. Rangan A, Handoll H, Brealey S, et al. Surgical vs nonsurgical treatment of adults with displaced fractures of the proximal humerus: the PROFHER randomized clinical trial. JAMA 2015; 313:1037-47.

4. Pawaskar AC, Lee KW, Kim JM, et al. Locking plate for proximal humeral fracture in the elderly population: serial change of neck shaft angle. Clin Orthop Surg 2012;4:209-15.

5. Calori GM, Colombo M, Bucci MS, et al. Complications in proximal humeral fractures. Injury 2016;47 Suppl 4:S54-8.

6. Boesmueller S, Wech M, Gregori M, et al. Risk factors for humeral head necrosis and non-union after plating in proximal humeral fractures. Injury 2016;47:350-5.

7. Brorson S, Bagger J, Sylvest A, Hrobjartsson A. Diagnosing displaced four-part fractures of the proximal humerus: a review of observer studies. Int Orthop 2009;33:323-7.

8. Papakonstantinou MK, Hart MJ, Farrugia R, et al. Interobserver agreement of Neer and AO classifications for proximal humeral 
fractures. ANZ J Surg 2016;86:280-4.

9. Bruinsma WE, Guitton TG, Warner JJ, Ring D; Science of Variation Group. Interobserver reliability of classification and characterization of proximal humeral fractures: a comparison of two and three-dimensional CT. J Bone Joint Surg Am 2013;95: 1600-4.

10. Sidor ML, Zuckerman JD, Lyon T, Koval K, Cuomo F, Schoenberg N. The Neer classification system for proximal humeral fractures: an assessment of interobserver reliability and intraobserver reproducibility. J Bone Joint Surg Am 1993;75:1745-50.

11. Brorson S, Olsen BS, Frich LH, et al. Surgeons agree more on treatment recommendations than on classification of proximal humeral fractures. BMC Musculoskelet Disord 2012;13:114.

12. Berkes MB, Dines JS, Little MT, et al. The impact of three-dimensional CT imaging on intraobserver and interobserver reliability of proximal humeral fracture classifications and treatment recommendations. J Bone Joint Surg Am 2014;96:1281-6.

13. Foroohar A, Tosti R, Richmond JM, Gaughan JP, Ilyas AM. Classification and treatment of proximal humerus fractures: inter-observer reliability and agreement across imaging modalities and experience. J Orthop Surg Res 2011;6:38.

14. Fleiss JL, Cuzick J. The reliability of dichotomous judgments: unequal numbers of judges per subject. Appl Psychol Meas 1979;3:537-42.

15. Fleiss JL, Levin B, Paik MC. Statistical methods for rates and proportions. 3rd ed. Hoboken (NJ): John Wiley \& Sons; 2013.

16. Gradl G, Knobe M, Pape HC, Neuhaus PV, Ring D, Guitton T. Decision making in displaced fractures of the proximal humerus: fracture or surgeon based. Int Orthop 2015;39:329-34.

17. Sjödén GO, Movin T, Güntner P, et al. Poor reproducibility of classification of proximal humeral fractures: additional CT of minor value. Acta Orthop Scand 1997;68:239-42.

18. Mutch J, Laflamme GY, Hagemeister N, Cikes A, Rouleau DM. A new morphological classification for greater tuberosity fractures of the proximal humerus: validation and clinical implications. Bone Joint J 2014;96:646-51.

19. Sukthankar AV, Leonello DT, Hertel RW, Ding GS, Sandow MJ. A comprehensive classification of proximal humeral fractures: HGLS system. J Shoulder Elbow Surg 2013;22:e1-6.

20. Bufquin T, Hersan A, Hubert L, Massin P. Reverse shoulder arthroplasty for the treatment of three- and four-part fractures of the proximal humerus in the elderly: a prospective review of 43 cases with a short-term follow-up. J Bone Joint Surg Br 2007;89: 516-20.

21. Chalmers PN, Slikker W 3rd, Mall NA, et al. Reverse total shoulder arthroplasty for acute proximal humeral fracture: comparison to open reduction-internal fixation and hemiarthroplasty. J Shoulder Elbow Surg 2014;23:197-204.

22. Cvetanovich GL, Chalmers PN, Verma NN, Nicholson GP, Romeo AA. Open reduction internal fixation has fewer short-term complications than shoulder arthroplasty for proximal humeral fractures. J Shoulder Elbow Surg 2016;25:624-31.

23. Gomberawalla MM, Miller BS, Coale RM, Bedi A, Gagnier JJ. Meta-analysis of joint preservation versus arthroplasty for the treatment of displaced 3- and 4-part fractures of the proximal humerus. Injury 2013;44:1532-9.

24. Okike K, Lee OC, Makanji H, Harris MB, Vrahas MS. Factors associated with the decision for operative versus non-operative treatment of displaced proximal humerus fractures in the elderly. Injury 2013;44:448-55.

25. Koval KJ, Gallagher MA, Marsicano JG, Cuomo F, McShinawy A, Zuckerman JD. Functional outcome after minimally displaced fractures of the proximal part of the humerus. J Bone Joint Surg Am 1997;79:203-7.

26. Elidrissi M, Bensaad S, Shimi M, Elibrahimi A, Elmrini A. Surgical treatment of proximal humeral fractures anatomical plate versus palm tree pinning (26 cases). Chir Main 2013;32:25-9.

27. Zhu Y, Lu Y, Shen J, Zhang J, Jiang C. Locking intramedullary nails and locking plates in the treatment of two-part proximal humeral surgical neck fractures: a prospective randomized trial with a minimum of three years of follow-up. J Bone Joint Surg Am 2011;93:159-68.

28. Sohn HS, Jeon YS, Lee J, Shin SJ. Clinical comparison between open plating and minimally invasive plate osteosynthesis for displaced proximal humeral fractures: a prospective randomized controlled trial. Injury 2017;48:1175-82.

29. Agarwal S, Rana A, Sharma RK. Functional outcome after primary hemiarthroplasty in three or four part proximal humerus fracture: a short term followup. Indian J Orthop 2016;50:590-4.

30. Liao W, Zhang H, Li Z, Li J. Is arthroscopic technique superior to open reduction internal fixation in the treatment of isolated displaced greater tuberosity fractures. Clin Orthop Relat Res 2016;474:1269-79. 